\title{
The effect of cervical mobilization on balance and static plantar loading distribution in patients with multiple sclerosis
}

\author{
A randomized crossover study
}

Tuba K. Maden, PhD, Kezban Y. Bayramlar, PhD, Yavuz Yakut, PhD.

\begin{abstract}

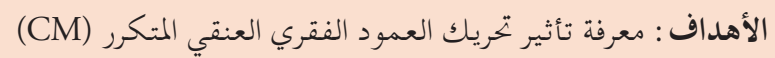

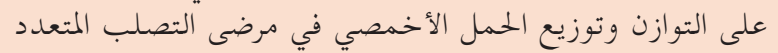

المنهجية : اشتملت هذه الدراسة على 12 فردا . أجريت خلال المبل الفترة

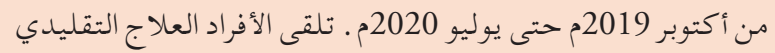

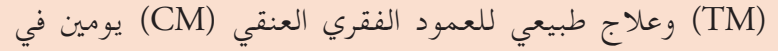

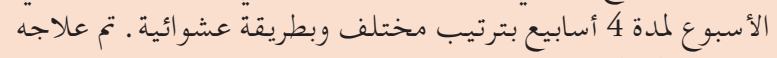

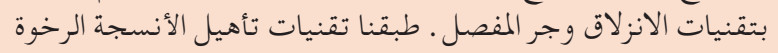

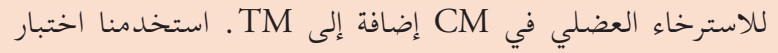

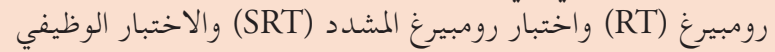

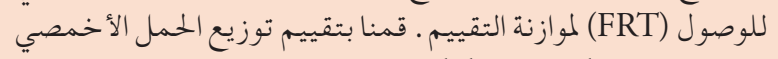

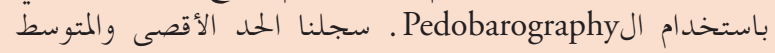

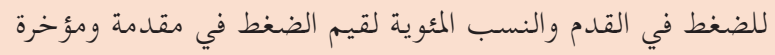

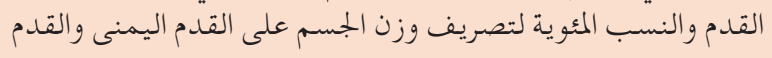

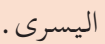

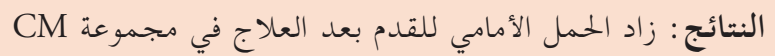

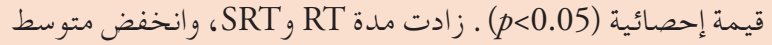

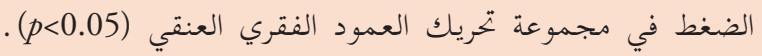

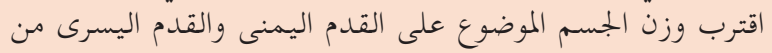

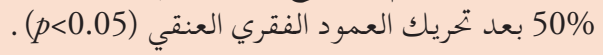

الخلاصة : يمكن لتقنيات تحريك العمود الفقري العنقي أن تغير

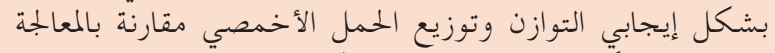

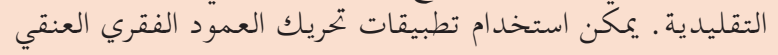

لدعم إعادة التأهيل العصبي استخدام تطبيق.

Objectives: To investigate the influence of repeated cervical mobilization $(\mathrm{CM})$ on balance and plantar loading distribution in patients with Multiple Sclerosis (MS).
Methods: A total of 12 individuals were included in the cross over study designed as a cross sectional. The study was carried out from October 2019 until July 2020. Individuals received traditional treatment (TM) and cervical mobilization treatments (CM) 2 days a week for 4 weeks in a different order by random method. It was treated with joint traction and sliding techniques. Soft tissue mobilization techniques for myofascial relaxation were applied for CM in addition to TM. Romberg test (RT), Sharpened Romberg Test (SRT), and Functional Reach Test (FRT) were used to balance the assessment. Plantar loading distribution was evaluated with Pedobarography. The maximum and mean pressure in the foot, the percentages of pressure values in the fore and rear of the foot, and percentages of the bodyweight discharge onto right feet and left feet were recorded.

Results: The forefoot loading increased after treatment in the CM group $(p<0.05)$. The duration of RT and SRT increased, and average pressure decreased in the cervical mobilization group $(p<0.05)$. The body weight discharge onto right feet and left feet approached $50 \%$ after cervical mobilization $(p<0.05)$.

Conclusion: Cervical mobilization techniques can positively change the balance and plantar loading distribution compared to traditional treatment. Cervical mobilization applications could be used to support neurological rehabilitation.

Neurosciences 2022; Vol. 27 (1):31-39 doi: 10.17712/nsj.2022.1.20210099

From the Department of Physiotherapy and Rehabilitation, Faculty of Health Sciences, Hasan Kalyoncu University, Gaziantep, Turkey

Received 2nd August 2021. Accepted 26th October 2021.

Address correspondence and reprint request to: Dr. Tuba K. Maden, Department of Physiotherapy and Rehabilitation, Faculty of Health Sciences, Hasan Kalyoncu University, Gaziantep, Turkey. E-mail: tuba.kaplan@hku.edu.tr

ORCID ID: https://orcid.org/0000-0001-8713-0825 
$\mathrm{M}$ ultiple sclerosis (MS) is a demyelinating, inflammatory, chronic, and neurodegenerative central nervous system (CNS) disease. ${ }^{1}$ Motor findings (spasticity, weakness), sensory findings (numbness, tingling, loss of sensation), balance and coordination problems, fatigue, visual dysfunction, cognitive disorder, bulbar symptoms (dysarthria, dysphagia respiratory problems), bladder-bowel dysfunction can be observed in patients. ${ }^{2}$ Studies show that $80 \%$ of patients have different levels of sensory impairment. ${ }^{3}$ The sense of proprioception, which is part of the somatosensory system, has an important role in the regulation of the vestibular system. This sense is obtained from the receptors in the joints, muscles, tendons, and ligaments and constitutes the position perception of the extremities and the body. Proprioceptors present in different proportions in different body structures and tissues are especially rich in the cervical region. ${ }^{4}$ Studies have proved that different treatment methods such as vibration and Kinesio taping on the cervical region stimulate the proprioceptive system, and these methods improve visual and vestibular connections..$^{5-7}$ Another method that can be applied to activate the proprioceptive system in this area is cervical mobilization techniques.

Manual techniques affect mechanotransduction, converting mechanical impulses into biological signals by myocytes and fibroblasts. ${ }^{8}$ Thus, silent gamma motor neurons are stimulated by inputs in muscle spindle afferents and smaller-diameter afferents. ${ }^{9}$ The cervical region is important for postural control, and cervical afferents provide input to cervicocollic, cervicooculler, and tonic neck reflex. So that postural stability, as well as head and eye movements, are controlled and maintained. ${ }^{10}$ Muscle spindles in the cervical region, especially in the suboccipital area are found in high densities. ${ }^{10}$ The application of manual therapy techniques to the cervical region increases motor control by increasing the proprioception transmitted from the vertebra segment to the central nervous system. ${ }^{11}$ These techniques also increase short-term motor neuron activity, resulting in increased performance in activities related to proprioception. ${ }^{12}$ It was known that the effects of mobilization techniques with neurophysiological effects on balance development, but studies investigating the effect of cervical mobilization

Disclosure. Authors have no conflict of interests, and the work was not supported or funded by any drug company. are limited to orthopedic cases or healthy persons. ${ }^{9,13}$

Moreover, many studies in literature focused that manual therapy techniques diminish pain, improve range of motion, quality of life in groups without neurological disorders. ${ }^{14-16}$ In other words, both the sample variety in terms of disorders and the treatment effects were examined in a narrow range.

The stimulation of the cervical region rich in proprioceptors increases somatosensory system activity so that it contributes to the improvement of balance. Balance and gait problems were frequently seen in MS patients. ${ }^{17}$ The foot, which carries the whole body weight and plays an important role in locomotion, provides a base of support for balance. ${ }^{18}$ Balance and plantar pressure were investigated together in our study since the sensorial inputs for balance are obtained from plantar pressure. In accord with the move, the base of support constantly changes during the walk, daily activities. As change base of support, plantar load distribution alters. ${ }^{18}$ Main plantar load on the forefoot has been associated with balance disorders and falls. ${ }^{19}$

An individual can provide balance by using toe pressure to correct the many postural disturbances that are experienced in everyday life. ${ }^{18}$ In addition, the plantar sensation obtained from the forefoot with the loading is essential in the control of balance. Moreover, it determined reduced peak pressure under the metatarsal head because of spasticity in MS. ${ }^{20}$ It is unknown how plantar pressure distribution changes with rehabilitation in MS patients.

Based on this background, the literature supports the positive effect of cervical mobilization in neurological disorders. However, only one study investigated the effect of cervical mobilization in MS. ${ }^{21}$ Therefore, our study is planned to investigate the effects of repeated cervical mobilization on balance and plantar pressure in MS individuals.

Methods. Study design. The study design was a randomized crossover study.

Ethics approval. The participants who were included in this study signed a volunteer consent form and all procedures were applied in accordance with the Declaration of Helsinki. The necessary approval for this study was received from Hasan Kalyoncu University, Non-invasive Research Ethics Committee on 01/10/2019 and with the decision no: 2019/0107. The study was conducted in Physiotherapy and Rehabilitation Research and Application Center between October 2019 and July 2020.

Participants. Expanded Disability Status Scale (EDSS) score is between 2-5, spasticity between 1 and 
Table 1 - Characteristics of individuals according to groups.

\begin{tabular}{|c|c|c|c|c|}
\hline Variables & Traditional rehabilitation first $(\mathrm{n}=5)$ & Cervical mobilization first $(\mathrm{n}=7)$ & $\mathrm{Z}$ & $P$-value \\
\hline Age (y) & $38.63 \pm 7.69$ & $36.00 \pm 8.56$ & 0.72 & 0.47 \\
\hline BMI $\left(\mathrm{kg} / \mathrm{m}^{2}\right)$ & $23.90 \pm 3.77$ & $24.91 \pm 3.94$ & 0.49 & 0.62 \\
\hline Disease duration $(\mathrm{y})$ & $9.63 \pm 5.12$ & $7.40 \pm 4.78$ & 0.88 & 0.38 \\
\hline EDSS score $(0-10)$ & $3.18 \pm 1.14$ & $2.27 \pm 1.00$ & 1.88 & 0.06 \\
\hline $\mathrm{RT}(\mathrm{sec})$ & $19.32 \pm 33.63$ & $25.87 \pm 34.06$ & 0.65 & 0.51 \\
\hline SRT (sec) & $9.95 \pm 8.48$ & $9.06 \pm 9.57$ & 0.16 & 0.87 \\
\hline FRT $(\mathrm{cm})$ & $24.09 \pm 4.91$ & $24.09 \pm 5.01$ & 1.81 & 0.07 \\
\hline
\end{tabular}

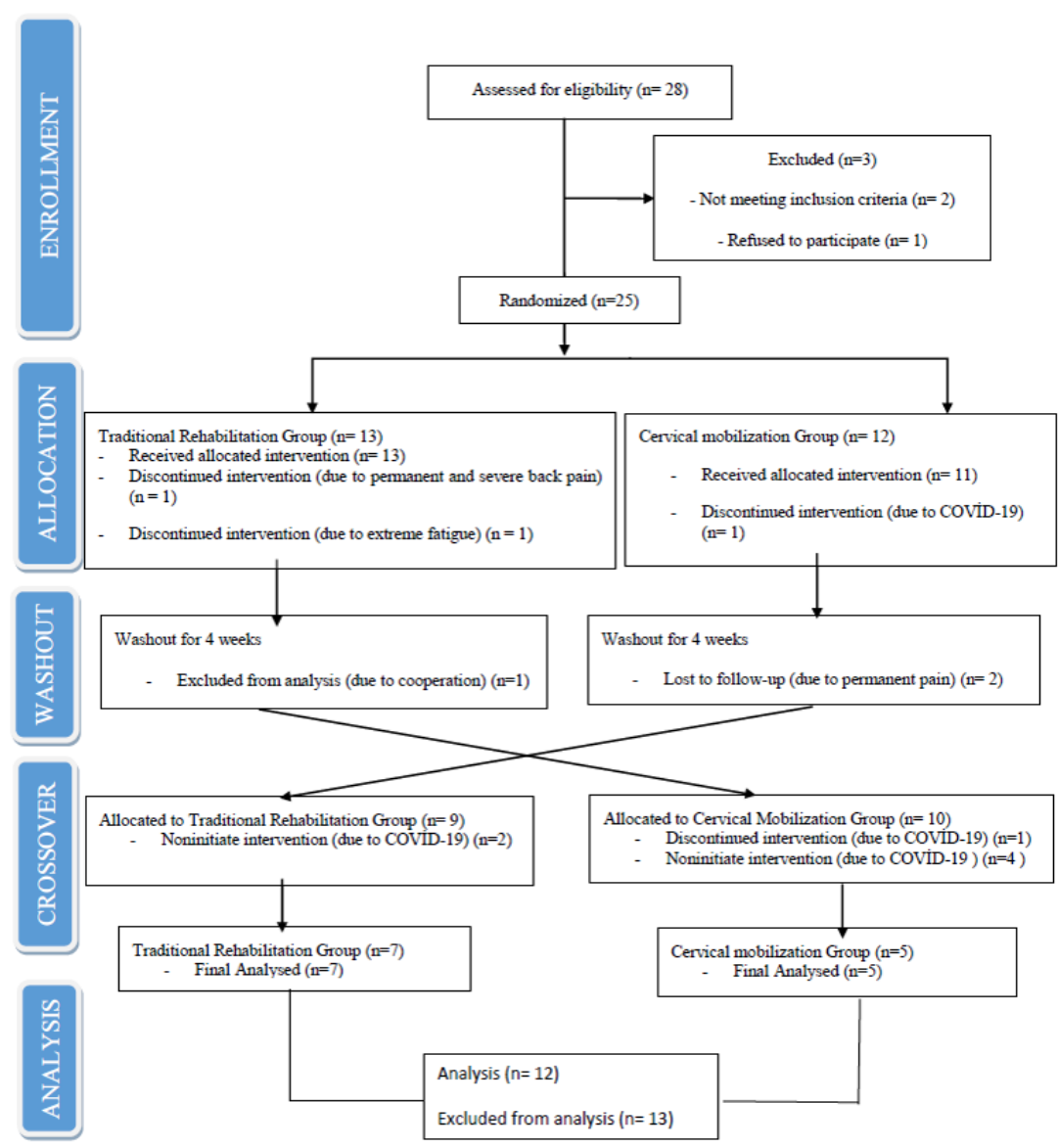

Figure 1 - CONSORT flowchart of the study.

3. According to the Modified Ashworth Scale (MAS), EDSS Cerebellar System Sub-Scale, Functional System Score is $>1$. The medical status is consistent, no drug alters in the last month, no orthopedic problems and no other neurological disorders to prevent involvement in this study were determined as the criteria for inclusion in this study. The exclusion criteria in the study were determined the presence of psychiatric or severe cognitive dysfunction, positive vertebro-bacillary test, positive cervical ligament instability tests (for Alar ligament and Transverse ligament), pregnancy, botulinum toxin application in the last 6 months, an attack in the last 3 months, physiotherapy program in the last 6 months.

A total of 28 individuals were recruited for this study. 3 individuals were excluded because they 
Table 2 - Pre-intervention and Post-intervention values for balance.

\begin{tabular}{|c|c|c|c|c|c|c|c|c|}
\hline \multirow[t]{2}{*}{ Variables } & \multicolumn{3}{|c|}{ Traditional Rehabilitation First $(n=5)$} & \multicolumn{3}{|c|}{ Cervical Mobilization First $(\mathrm{n}=7)$} & \multirow{2}{*}{$\begin{array}{c}\text { Period effect } \\
P \text {-value }\end{array}$} & \multirow{2}{*}{$\begin{array}{c}\text { Carryover effect } \\
P \text {-value }\end{array}$} \\
\hline & Pre-intervention & Post-intervention & $P$-value & Pre-intervention & Post-intervention & $P$-value & & \\
\hline RT & $19.32 \pm 33.63$ & $31.03 \pm 26.48$ & $0.04^{*}$ & $25.87 \pm 34.06$ & $49.94 \pm 47.54$ & $0.03^{*}$ & 0.06 & 0.94 \\
\hline SRT & $9.95 \pm 8.48$ & $18.29 \pm 10.22$ & $0.00^{*}$ & $9.06 \pm 9.57$ & $10.49 \pm 11.51$ & $0<01^{*}$ & 0.09 & 0.31 \\
\hline FRT & $24.90 \pm 4.91$ & $28.28 \pm 4.06$ & $0.00^{*}$ & $24.09 \pm 5.01$ & $29.42 \pm 6.49$ & $0<01^{*}$ & 0.71 & 0.65 \\
\hline
\end{tabular}

Table 3 - Pre-intervention and Post-intervention values for plantar pressure.

\begin{tabular}{|c|c|c|c|c|c|c|c|c|}
\hline \multirow[t]{2}{*}{ Variables } & \multicolumn{3}{|c|}{ Traditional Rehabilitation First } & \multicolumn{3}{|c|}{ Cervical Mobilization First } & \multirow{2}{*}{$\begin{array}{c}\text { Period effect } \\
P \text {-value }\end{array}$} & \multirow{2}{*}{$\begin{array}{c}\text { Carryover effect } \\
P \text {-value }\end{array}$} \\
\hline & Pre-intervention & Post-intervention & $P$-value & Pre-intervention & Post-intervention & $P$-value & & \\
\hline Maxipress & $558.68 \pm 98.52$ & $581.87 \pm 195.66$ & 0.57 & $578.25 \pm 89.11$ & $536.43 \pm 72.62$ & 0.24 & 0.91 & 0.33 \\
\hline Averagepress & $250.86 \pm 47.41$ & $254.54 \pm 64.77$ & 0.20 & $302.42 \pm 63.97$ & $251.79 \pm 23.29$ & $0.01^{*}$ & 0.87 & 0.53 \\
\hline Dforeper & $37.53 \pm 5.35$ & $37.02 \pm 8.65$ & 0.26 & $36.85 \pm 6.11$ & $39.78 \pm 7.48$ & $0.02^{*}$ & 0.11 & 0.65 \\
\hline NDforeper & $35.78 \pm 7.71$ & $35.19 \pm 8.07$ & 0.77 & $44.27 \pm 7.92$ & $42.70 \pm 8.17$ & 0.44 & 0.07 & 0.25 \\
\hline Drearper & $62.46 \pm 5.35$ & $62.98 \pm 8.65$ & 0.26 & $63.14 \pm 6.11$ & $60.21 \pm 7.48$ & $0.02^{*}$ & 0.11 & 0.69 \\
\hline NDrearper & $64.21 \pm 7.71$ & $64.81 \pm 8.07$ & 0.77 & $55.72 \pm 7.92$ & $57.29 \pm 8.17$ & 0.44 & 0.07 & 0.25 \\
\hline Dloadper-tot & $53.14 \pm 4.25$ & $52.25 \pm 6.31$ & 0.55 & $52.05 \pm 2.60$ & $52.37 \pm 3.16$ & 0.92 & 0.58 & 0.88 \\
\hline NDloadper-tot & $46.85 \pm 4.25$ & $47.75 \pm 6.31$ & 0.55 & $47.94 \pm 2.60$ & $47.62 \pm 3.16$ & 0.92 & 0.58 & 0.87 \\
\hline $\begin{array}{l}\text { Maxipress - } \\
\text { nondominan } \\
\text { pressure in rea }\end{array}$ & $\begin{array}{l}\text { num pressure } \\
\text { entages of press } \\
\text { Dloadper-tot }\end{array}$ & $\begin{array}{l}\text { Averagepress } \\
\text { forefoot, Drea } \\
\text { entages of total }\end{array}$ & 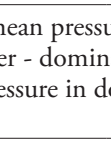 & $\begin{array}{l}\text { of foot, Dforepe } \\
\text { it-percentages of } \\
\text { ninant foot, NDl } \\
p<0.05^{*}\end{array}$ & $\begin{array}{l}\text { ominant-perce } \\
\text { ure in rearfoot } \\
\text { er-tot - percen }\end{array}$ & 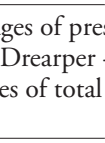 & $\begin{array}{l}\text { e in for } \\
\text { ndomir }\end{array}$ & $\begin{array}{l}\text { NDforeper - } \\
\text { sercentages of } \\
\text { lominant foot, }\end{array}$ \\
\hline
\end{tabular}

rejected to enrol or did not meet the inclusion criteria. Eight individuals discontinued treatment because of COVID-19. Since one individual has extreme fatigue, one individual has co-operation, one individual has severe back pain, 2 individuals have permanent pain. CONSORT Flowchart was given in Figure 1. The TM group included a total of 5 individuals ( 5 females) with a mean age of $38.64 \pm 7.69$ years, CM group included a total of 7 individuals ( 1 male and 6 females) with a average age of $36.00 \pm 8.56$ years. The study was completed with 12 of the individuals included.

Procedure and randomization. After screening, individuals were grouped according to the closed envelope method of randomization and stratified by age, EDSS score, gender to receive either a TM or CM 4 -week crossover intervention. This study was singleblind. The person who produced statistical analyses did not know the group in which they were included. To avoid any interference between the 2 interventions, all subjects rested 4 weeks before initiating the another intervention. Individuals in TM took firstly traditional rehabilitation, secondly cervical mobilization after washout period. Individuals in CM took firstly cervical mobilization, secondly traditional mobilization after washout period. During the 4-week washout period, the subjects rested without treatment or home exercise. The same examiner administered both balance and plantar pressure analyses before and after each 4-week treatment. Four analyses were carried out for each individual. The first analyses were done at baseline, and the second analyses were done when the first treatment was completed. The third analyses were done when the washout period was over at the beginning of the second treatment, and the fourth was done when two treatments were over. All of the assessments and treatments were carried out by the same physiotherapist. All of the assessments and treatments were provided individually and face-to-face.

The EDSS was used for neurological impairment and degree of disability in multiple sclerosis patients. EDSS has a score ranging from 0 (normal neurological findings) to 10 (death from MS). This scale is a valid and reliable measure of disability and impairment in individuals with MS. ${ }^{22}$

Romberg, Sharpened Romberg were performed, and the duration of the tests was recorded. Romberg test (RT) was carried out the individuals with feet together and eyes closed. When the individual held it in one place, oscillations started, or was likely to fall, the test was lasted. ${ }^{23}$ Romberg test's maximum time was 120 
sec. The RT is clinically used for vestibular disorders and problems. ${ }^{23}$ Sharpened Romberg (SR) was applied on a straight line with one leg behind the other leg, eyes open, without impairing the balance and leaving the arms sagging. The time end criteria were defined as the time that took a individual to displace the foot, contact the observer to avoid falling, and reach the maximal duration of $30 \mathrm{sec}^{24}$ The validity and reliability of SR were shown in a different population that includes persons with neurological disorders and older adults. ${ }^{25}$

Functional Reach Test (FRT) was used for assess individuals' dynamic balance. Individuals had wanted their hands at 90 degrees by conservation the dominant arms' elbow straight on the wall. The test was applied as reaching onward without taking a step and losing the balance on the wall. The metacarpal bone level was signed in the beginning position and finishing position. ${ }^{26}$ The difference between these levels was measured. ${ }^{27}$ The test was repeated 3 times, and the average was recorded.

A pedobarography system was used for pressure analyses and plantar loading. The Pedobarography system comprises color printer, a screen, remote control device, pressure sensor platform, printer-to-platform, power unit, and screen-to-platform connections. This device's pressure measuring platform is in $650 \times 300 \times 25$ $\mathrm{mm}$ size, and the platform contains a 360x180 mm sensor area, three sensors per $\mathrm{cm}^{2}$. The specifications of static pedobarography detailed as follow: the sampling rate of the device is 14 frames per second, storage range is 18 frames, pressure range $2-127 \mathrm{~N} / \mathrm{cm}^{2}$, resolution $1 \mathrm{~N} / \mathrm{cm}^{2}$, accuracy percentage $5 \%$ depending on the foot, the temperature range in measurements $15-40{ }^{\circ} \mathrm{C}$, connection power is $220 / 110$ volts. During the static measurements, the subjects were distracted by the questions asked so that individuals do not direct their body weight to a certain side on the platform. For an accurate assessment, they were requested to look at a fixed point on the wall three meters ahead. The evaluation was made separately for both feet (dominant and nondominant sides). The mean and maximum pressure, the ratios of pressure values in the rear and fore of the foot, ratios of the total pressure falling to the foot were evaluated.

Rehabilitation program. The TM program was contained stretching for the lower limbs in all patients, coordination and balance exercises according to the patient's grade, strengthening exercises for the muscles required. Individuals participated in 2 training sessions per week for four weeks. Each training session comprised of a 10-minute stretching, a 30-minute balance and strengthening exercise, 5-minute non-balance coordination exercise.
During the CM program, cervical mobilization techniques were carried to the patients for 30 minutes in addition to the TM program. In the supine position, the patients carried out general and segmental traction and suboccipital relaxation to the cervical spine at the start of treatment. Myofascial relaxation technique was applied to the levator scapulae, scalen muscles in the supine position, and the trapezius muscle in the side-lying position (Appendices 1). Segmental rotational mobilization was performed in a sitting position. Mobilizations were performed at Grade I-II level according to the Kaltenborn technique. The mobilization and relaxation techniques were started with five repetitions, and the number of repetitions was increased to 10 repetitions.

Statistical analysis. Statistical Package for the Social Sciences version 22.0 software was used for descriptive statistical analysis. The frequency in percent (\%) and mean \pm standard deviation $($ mean \pm SD) of necessary variables were calculated for the descriptive analyses. Shapiro-Wilk test was used to examine the normality of the data. Mann Whitney-U test was used for investigate the homogeneity between TM and CM. Wilcoxon signed-rank test was used in the comparison of the two measuring conditions; before and after treatments. The carryover effect and period effect was evaluated with NCSS 2020 (Statistical Software, 2020; NCSS, LLC, Kaysville, Utah, USA). The period effect was the importance of how individuals receive treatments and compare the groups' 4th evaluations. The carryout effect was whether the first treatment's effect continues when starting the second treatment and is determined by comparing the 1 st and 3rd evaluation of a group for long-term effect. The groups were combined since it was seen that there was no carryout effect and period effect in all data. Wilcoxon signed-rank test was used to comparison traditional treatment and cervical mobilization treatment in all individuals. In all findings, the significance value was accepted as $p<0.05$. G*Power application was used for post-hoc power analysis. The post-hoc power analysis was calculated $\% 96$ with 2 tails, 0.05 Type I error, and effect size 0.44 in accordance with RT.

Results. The distribution of individuals' characteristics, according to groups, is shown in Table 1. There were no statistically significant differences in body mass index, age, disease duration, EDSS score, and balance scores between the traditional and cervical mobilization groups ( $p>0.05)$.

The distribution of balance was shown in Table 2, according to pre-intervention and post-intervention. After treatment, static and dynamic balance scores were increased 
Table 4 - Comparison of traditional rehabilitation and cervical mobilization in all individuals.

\begin{tabular}{|c|c|c|c|c|c|c|c|c|}
\hline \multirow[t]{2}{*}{ Variables } & \multicolumn{4}{|c|}{ Traditional rehabilitation $(\mathrm{n}=12)$} & \multicolumn{4}{|c|}{ Cervical mobilization $(n=12)$} \\
\hline & Pre-intervention & Post-intervention & $\mathrm{Z}$ & $p$ & Pre-intervention & Post-intervention & $\mathrm{Z}$ & $p$ \\
\hline RT & $40.71 \pm 51.96$ & $46.68 \pm 41.37$ & 1.33 & 0.18 & $44.12 \pm 50.30$ & $66.75 \pm 52.37$ & 2.54 & $0.01^{*}$ \\
\hline SRT & $13.90 \pm 11.20$ & $19.83 \pm 10.83$ & 2.93 & $0.00^{*}$ & $14.64 \pm 11.70$ & $21.85 \pm 10.43$ & 2.49 & $0.01^{*}$ \\
\hline FRT & $28.86 \pm 3.58$ & $30.35 \pm 3.46$ & 0.80 & 0.42 & $26.17 \pm 5.17$ & $29.88 \pm 5.94$ & 1.88 & 0.06 \\
\hline Maxipress & $570.36 \pm 105.70$ & $604.17 \pm 170.91$ & 1.17 & 0.85 & $592.80 \pm 116.32$ & $546.37 \pm 89.88$ & 1.36 & 0.17 \\
\hline Averagepress & $253.49 \pm 49.29$ & $272.33 \pm 55.91$ & 0.53 & 0.59 & $285.93 \pm 60.39$ & $253.10 \pm 37.62$ & 2.69 & $0.00^{*}$ \\
\hline Dforeper & $37.36 \pm 5.82$ & $36.53 \pm 8.32$ & 0.00 & 1.00 & $36.73 \pm 7.75$ & $37.72 \pm 7.86$ & 0.31 & 0.75 \\
\hline NDforeper & $40.35 \pm 6.46$ & $38.33 \pm 6.12$ & 0.44 & 0.65 & $40.55 \pm 8.12$ & $40.30 \pm 9.93$ & 0.07 & 0.94 \\
\hline Drearper & $62.36 \pm 5.82$ & $63.46 \pm 8.32$ & 0.00 & 1.00 & $63.26 \pm 7.75$ & $62.27 \pm 7.86$ & 0.31 & 0.75 \\
\hline NDrearper & $59.64 \pm 6.46$ & $61.66 \pm 6.12$ & 0.44 & 0.65 & $59.44 \pm 8.12$ & $59.69 \pm 9.93$ & 0.70 & 0.94 \\
\hline Dloadper-tot & $53.76 \pm 2.32$ & $53.40 \pm 3.73$ & 0.35 & 0.72 & $53.41 \pm 2.65$ & $52.11 \pm 2.79$ & 2.13 & $0.03^{*}$ \\
\hline NDloadper-tot & $46.23 \pm 2.32$ & $46.60 \pm 3.73$ & 0.35 & 0.72 & $46.58 \pm 2.65$ & $47.88 \pm 2.79$ & 2.13 & $0.03^{*}$ \\
\hline
\end{tabular}

RT - Romberg Test, SRT - Sharpened Romberg Test, FTR - Functional Reach Test, Maxipress - maxiumum pressure of foot, averagepress - mean pressure of foot, Dforeper - dominant-percentages of pressure in forefoot, NDforeper - nondominant-percentages of pressure in forefoot, Drearper - dominant-percentages of pressure in rearfoot, NDrearper - nondominant-percentages of pressure in rearfoot, Dloadper-tot - percentages of total pressure in dominant foot, NDloadper-tot - percentages of total pressure in nondominant foot, $p<0.05$

in both groups $(p<0.05)$ (Table 2$)$. 4 weeks was sufficient to clear the effect of the treatments applied to both groups on balance $(p>0.05)$ (Table 2$)$.

Pre-intervention and post-intervention values for plantar pressure were shown in Table 3. There was no difference in plantar pressure distribution in the traditional treatment group. The average pressure and rearfoot loading significantly decreased after treatment in the CM group $(p<0.05)$ (Table 3 ). In addition to this, forefoot loading increased after treatment in the CM group $(p<0.05)$ (Table 3). There was no difference between the pre-intervention evaluations, and the carryover effect was not seen at the mean pressure $(p>0.05)$.

To compare traditional treatment's and cervical mobilization treatment's effect in all individuals, RT's and SRT's duration increased, and an average of pressure decreased in the cervical mobilization group $(p<0.05)$ (Table 4). The body weight discharge onto the right feet and left feet approached $50 \%$ after cervical mobilization $(p<0.05)$ (Table 4).

Discussion. In our study, investigating the effects of cervical mobilization on balance and plantar pressure in MS patients, cervical mobilization can positively change the balance and plantar loading distribution compared to traditional treatment. This study is the investigating the repeated effect of cervical mobilization in neurological diseases. It was determined that the treatments performed had no long-term effects for each parameter in this study.
In a thesis study, the immediate effect of cervical mobilization on balance in MS patients was investigated. Balance assessment was performed with posturography in the thesis study, and a significant improvement was observed in the stability limit test. ${ }^{21} \mathrm{It}$ shows that cervical techniques stimulate proprioceptors and decrease gama motor neuron activity, so techniques improve balance and regulate spasticity. Comparison methods, the techniques in the thesis were included in our method, and our applications were made more comprehensive and more extended in each session. Our techniques were repeatedly applied for 2 sessions per week as a protocol, and a traditional treatment group was included in this study. Some studies investigate the influence of cervical mobilization techniques on balance and fall in older adults or healthy persons. ${ }^{13,28}$ Three different spinal manipulation methods were performed on 186 individuals with neck pain, and the balances of the individuals were compared. ${ }^{29}$ While upper cervical mobilization (C1-2) was applied to one group, a combination of thoracic (T5-6), middle cervical (C3-4), cervicothoracic joint (C7-T1) mobilizations were applied to the other group. The stabiliometric measurements used for balance were examined. While there was no change in the combined mobilization group's postural oscillations, balance improved in the upper cervical vertebra group. When the balance was evaluated 15 days after the treatment, it was observed 
that the effect of the treatment still continued in the upper cervical mobilization group. ${ }^{29}$ Smith et al. show that mobilizations applied to the upper cervical vertebra made a positive difference in the Romberg test on healthy individuals. ${ }^{30}$ In another study similar to this, individuals have performed occipito-atlantoaxial spinal mobilization. The treatment's effectiveness was proven by the displacement of the center of body mass. ${ }^{31}$ Considering the neurophysiological effects of the applications on the cervical region, we think that these applications can be a treatment option for individuals with orthopedic problems and individuals with neurological diseases such as stroke, ataxia, and Parkinson's balance and gait problems. In our study for this purpose, the manual therapy methods we applied to the cervical region included not only the upper cervical vertebra but also myofascial relaxation techniques for all cervical segments and muscles in the suboccipital region. Our study showed that cervical mobilization application could improve balance. We think that the treatment protocol applied can be recommended to patients.

The cervical region, whose importance is understood in terms of sensory and vestibular reflexes, is a topic in the literature where different methods are applied. The effect of these methods is investigated. One of these methods is the application of segmental vibration. Leplaideur et $\mathrm{al}^{6}$ gave vibration to neck muscles on the hemiplegic side and assessed the treatment's immediate effect. They recorded an improvement in postural asymmetry, body image, and balance in hemiplegic individuals. ${ }^{5}$ Balance results after segmental vibration therapy applied to the cervical region in Parkinson's patients were similar to the healthy control group.

Perenneou et al. applied transcutaneal electrical stimulation to the neck muscles in hemiplegic patients and proved that treatment reduced instability in the sitting position. ${ }^{32}$ In a study in which the balance of hemiplegic patients was examined by providing proprioceptive training for the neck region, the balance of individuals improved. ${ }^{33}$ Another treatment applied to the neck area is the application of Kinesio taping. The efficiency of taping for the paraspinal muscles in the neck region on 23 individuals was investigated using the Y balance test, and it was shown that balance improved in individuals. ${ }^{7}$ These studies' effect mechanism on balance is the same neurological mechanism, which explains the effect of cervical mobilization on balance. The findings of our study on balance are compatible with the literature, and the balance has improved. However, our balance assessments were measured by clinical tools. Considering the innovative aspect of our study, our results on balance should be supported by detailed evaluations.

Balance and falls have been associated with the main plantar load in the foot. ${ }^{19}$ Metatarsal heads pressure is used to compensate for postural perturbations. ${ }^{18}$ While this pressure on the forefoot is an important component for controlling balance, it creates the anterior arc sense. Merying et $\mathrm{a}^{34}$ shown that peak pressure under the 3rd and 5th metatarsal heads was significantly reduced in hemiparetic patients than in the control group. Moreover, the same study determined that peak pressure under metatarsal heads load decreased as an increase of spasticity. In addition to this, it determined that reduced peak pressure under metatarsal head because of spasticity in MS. ${ }^{20}$ Mao et $\mathrm{al}^{18}$ investigated the effect of Tai-chi exercises on plantar pressure distribution and forefoot loading increased after training. In our study, cervical mobilization techniques, in addition to traditional treatment, increased forefoot loading, and decreased rearfoot loading in MS. Since there are specific connections between the suboccipital muscles and the central nervous system, we think that the plantar pressure distribution changes with the improvement of balance. ${ }^{10}$ Also, the decrease in the average of plantar pressure can be explained by the increase in the contact surface due to the improvement of balance. As developed in balance, trunk symmetry is improved, and the bodyweight discharge onto feet is equal. ${ }^{35}$ In accord with this, the bodyweight discharge onto right feet and left feet approached $50 \%$ after cervical mobilization in our study. The distribution of body weight on foot is formed coronal balance, and it is related to lateral pelvic tilt and postural alignment. ${ }^{36}$ Considering the neurophysiological effects of cervical mobilization, we think that mobilizations were active proprioceptors to improve the sense of position and alignment. In addition to this, the improved balance might have provided that the plantar load distribution was equal in the right and left feet.

In light of the results obtained in this study, cervical mobilization techniques effective on balance and plantar load distribution. The Physiotherapist can perform cervical mobilizations as a supportive application in a patient with multiple sclerosis.

The study has several limitations. One of them was a small number of patients. The effect of cervical mobilization on balance could be researched in detail. In the present study, only static plantar pressure parameters of the foot were evaluated, and the number of areas for foot pressure was limited.

Our study provides information that cervical mobilization techniques improve balance and change plantar load distribution in multiple sclerosis. More 
studies are needed that understand the effect of cervical mobilization in neurological disease. The effects of the cervical mobilization on alignment, posture, Tonus, and position sense should be investigated in neurological disease. The long-term effects of cervical mobilization should be examined.

In conclusions, this study provides evidence that cervical mobilization techniques can positively change the balance and plantar loading distribution compared to traditional treatment. Cervical mobilization applications could be used to support neurological rehabilitation.

Acknowledgement. The authors would like to to thank Scribendi for English language editing.

\section{References}

1. Frohman EM, Racke MK, Raine CS. Multiple sclerosis-the plaque and its pathogenesis. NEnglJ Med 2006; 354: 942-955.

2. Thompson AJ. Symptomatic management and rehabilitation in multiple sclerosis. J Neurol Neurosurg Psychiatry 2001; 71: ii22-ii27.

3. Leocani L, Martinelli V, Natali-Sora MG, Rovaris M, Comi G. Somatosensory evoked potentials and sensory involvement in multiple sclerosis: comparison with clinical findings and quantitative sensory tests. Mult Scler 2003; 9: 275-279.

4. Boyd-Clark L, Briggs C, Galea M. Muscle spindle distribution, morphology, and density in longus colli and multifidus muscles of the cervical spine. Spine 2002; 27:694-701.

5. Leplaideur S, Leblong E, Jamal K, Rousseau C, Raillon AM, Coignard P, et al. Short-term effect of neck muscle vibration on postural disturbances in stroke patients. Exp Brain Res 2016; 234: 2643-2651.

6. Kammermeier S, Dietrich L, Maierbeck K, Plate A, Lorenzl S, Singh A, et al. Neck vibration proprioceptive postural response intact in progressive supranuclear palsy unlike idiopathic Parkinson's disease. Front Neurol 2017; 8: 689.

7. Jeon YJ, Kim GM. Effects of Kinesio Taping on Craniovertebral Angle and Balance Ability in Subject with Forward Head Posture. Journal of the Korea Society of Computer and Information 2020; 25: 145-150.

8. Lederman E, editor. The science \& practice of manual therapy. 2nd Edition. Elsevier Health Sciences; 2005.

9. Pickar JG. Neurophysiological effects of spinal manipulation. Spine J 2002; 2: 357-371.

10. Treleaven JJ. Sensorimotor disturbances in neck disorders affecting postural stability, head and eye movement control. Man Ther 2008; 13: 2-11.

11. Fryer G. Intervertebral dysfunction: a discussion of the manipulable spinal lesion. Int J Osteopath Med 2003; 6: 64-73.

12. Jones D. The effect of osteopathic manipulative therapy applied to the lumbar spine on postural stability: a pilot study. Victoria: Victoria university: School of Health Sciences; 2004. Master Thesis.

13. Holt KR, Haavik H, Elley CR. The effects of manual therapy on balance and falls: a systematic review. J Manipulative Physiol Ther 2012; 35: 227-234.

14. Ho C-YC, Sole G, Munn J. The effectiveness of manual therapy in the management of musculoskeletal disorders of the shoulder: A systematic review. Man Ther 2009; 14: 463-474.

15. Clar C, Tsertsvadze A, Court R, Hundt GL, Clarke A, Sutcliffe P. Clinical effectiveness of manual therapy for the management of musculoskeletal and non-musculoskeletal conditions: systematic review and update of UK evidence report. Chiropr Man Therap 2014; 22: 12.

16. Voogt L, de Vries J, Meeus M, Struyf F, Meuffels D, Nijs J. Analgesic effects of manual therapy in patients with musculoskeletal pain: A systematic review. Man Ther 2015; 20 : 250-256.

17. Kheradmand M, Afshari M, Nasehi MM, Aghaei I, Shabani M, Farshidi F, et al. Prevalence of subtypes of multiple sclerosis and the most common clinical symptoms in Iranian patients: A meta-analysis. Clin Exp Neuroimmunol. 2019; 10: 33-40.

18. Mao D, Li J, Hong YJ. The duration and plantar pressure distribution during one-leg stance in Tai Chi exercise. Clin Biomech (Bristol, Avon) 2006; 21: 640-645.

19. Wu G, Hitt J. Ground contact characteristics of Tai Chi gait. Gait Posture 2005; 22: 32-39.

20. Femery V, Moretto P, Renaut H, Thévenon A, Lensel G. Measurement of plantar pressure distribution in hemiplegic children: changes to adaptative gait patterns in accordance with deficiency. Clin Biomech (Bristol, Avon) 2002; 17: 406-413.

21. Karanfil E. Multipl Sklerozlu Bireylerde Servikal Mobilizasyonun Spastisite ve Denge Üzerine Etkisi. Ankara: Hacettepe University: Health Sciences Institute; 2018. Master Thesis.

22. Kurtzke JFJN. Rating neurologic impairment in multiple sclerosis: an expanded disability status scale (EDSS). Neurology 1983; 33: 1444-1452.

23. Khasnis A, Gokula R. Romberg's test. J Postgrad Med 2003; 49: 169-172.

24. Widener GL, Allen DD, Gibson-Horn C. Randomized clinical trial of balance-based torso weighting for improving upright mobility in people with multiple sclerosis. Neurorehabil Neural Repair 2009; 23: 784-791.

25. Gras LZ, Ganley KJ, Bosch PR, Mayer JE, Pohl PSJP. Convergent validity of the Sharpened Romberg. Phys Occup Ther Geriatr. 2017; 35: 99-108.

26. Duncan PW, Weiner DK, Chandler J, Studenski SJ. Functional reach: a new clinical measure of balance. J Gerontol 1990; 45: M192-M197.

27. Schuhfried O, Mittermaier C, Jovanovic T, Pieber K, Paternostro-Sluga T. Effects of whole-body vibration in patients with multiple sclerosis: a pilot study. Clin Rehabil 2005; 19: 834-842.

28. Kendall JC, Hartvigsen J, French SD, Azari MF. Is there a role for neck manipulation in elderly falls prevention? - An overview. J Can Chiropr Assoc 2015; 59: 53-63.

29. Romero del Rey R, Saavedra Hernández M, Rodríguez Blanco C, Palomeque del Cerro L, Alarcón Rodríguez R. Short-term effects of spinal thrust joint manipulation on postural sway in patients with chronic mechanical neck pain: a randomized controlled trial. Disabil Rehabil 2020; 1-7.

30. Smith L, Mehta MJ. The effects of upper cervical complex 
high velocity low amplitude thrust technique and sub-occipital muscle group inhibition techniques on standing balance. Int J Osteopath Med. 2008; 11: 162.

31. Rico JMB. Evaluation and analysis of the influence of the suboccipital inhibition technique and the great abdominal maneuver on postural statics: baropodometric and stabilometric study: University of Cádiz; 2011

32. Perennou D, Pélissier J, Amblard B, editors. Posture and postural control following a cerebrovascular accident: a review. Ann Med Phys (Lille) 1996; 8: 497-513.

33. Kim G-M, Oh D-WJ. Neck proprioceptive training for balance function in patients with chronic poststroke hemiparesis: a case series. J Phys Ther Sci 2014; 26: 1657-1659.

34. Meyring S, Diehl RR, Milani TL, Hennig EM, Berlit P.
Dynamic plantar pressure distribution measurements in hemiparetic patients. Clin Biomech (Bristol, Avon) 1997; 12: 60-65.

35. Haumont T, Gauchard GC, Lascombes P, Perrin P. Postural instability in early-stage idiopathic scoliosis in adolescent girls.

Spine (Phila Pa 1976) 2011; 36: E847-54.

36. Ma Q, Lin H, Wang L, Zhao L, Chen M, Wang S, et al. Correlation between spinal coronal balance and static baropodometry in children with adolescent idiopathic scoliosis.

Gait Posture 2020; 75: 93-97.

\section{Appendices 1 -}

\begin{tabular}{ll}
\hline Technique & Description of technique \\
\hline General Traction in CM & $\begin{array}{l}\text { The patient was supine, and whereas the Physiotherapist was seated, the Physiotherapist's hands grasped } \\
\text { the patient's head. The force was applied with hands over the occiput in the ceiling direction with slight } \\
\text { traction in the cranial direction. }\end{array}$
\end{tabular}

Segmental Traction in CM

The patient was supine and whereas the Physiotherapist was seated. The Physiotherapist's hands fixed the inferior vertebra, and the force was applied with the superior vertebra in the direction of the ceiling with slight traction in the cranial direction.

The patient was supine, and whereas the Physiotherapist was seated, the patient's head with the elbows

Suboccipital Relaxation in CM resting on the surface of the table. The Physiotherapist's fingers flexed, and finger pads positioned on the posterior arch of the atlas to allow the occiput to rest in the palm of hands. A force was applied with the

finger pads over the atlas in the direction of the ceiling with slight traction in the cranial direction.

Myofascial Relaxation for Levator Scapula in CM

Myofascial Relaxation for Trapezius in CM

Myofascial Relaxation for Scalenius in CM

Segmental rotation mobilization in CM

Non-balance coordination exercise in TM

Balance exercises in TM

Strengthening exercises in TM

Stretching exercises in TM
The patient was supine. The Physiotherapist was standing at the edge of the table. Physiotherapist used to active release technique. The ischemic compression is applied in the middle of the muscle during the muscle stretching.

The patient was side-lying. The Physiotherapist was standing at the edge of the table. Physiotherapist used to active release technique. The ischemic compression is applied in the middle of the muscle during the muscle stretching.

The patient was supine. The Physiotherapist was standing at the edge of the table. Physiotherapist used to active release technique. The ischemic compression is applied in the middle of the muscle during the muscle stretching.

The patient was sitting and whereas the Physiotherapist was standing. The Physiotherapist's hands grasped the patient's head, and the inferior vertebra was fixed. The superior vertebra was rotated to the right and left direction by the Physiotherapist.

The Physiotherapist was standing. The patient was asked to make voluntary movements for the upper and lower extremities on different grounds (stable and unstable surface etc.)

The Physiotherapist was standing to ensure safety. Static and dynamic balance exercises were trained on different sizes of the support surface and different surfaces.

The Physiotherapist was standing. Therabants were used to strengthen upper extremity muscles. The exercises were performed on different surfaces like bad, Bobath ball. The exercises were chosen according to the individual's level.

The patient was supine. The Physiotherapist was standing. Gastrocnemius, Hamstring and adductor muscles were stretched by Physiotherapist. 